\title{
Stepwise Development and Yearlong Assessment of a Pharmacist-Driven Molecular Rapid Diagnostic Test Result Service for Bloodstream Infections
}

Andrew Ticcioni, PharmD ${ }^{1}$; Kyle Piscitello, PharmD 2; Matthew Bjornstad, PharmD ${ }^{3}$; Katie Hensley, PharmD ${ }^{4}$; Jim Davis, BPharm²; Annette Drobac, PharmD $D^{1}$; John Canepa, BPharm ${ }^{1}$

${ }^{1}$ Ascension Columbia St. Mary's Hospital; ${ }^{2}$ Ascension Wisconsin; ${ }^{3}$ Regions Hospital; ${ }^{4}$ Concordia University Wisconsin School of Pharmacy

\section{ABSTRACT}

Purpose: Provide a stepwise approach to the design and implementation of a service that integrates all staff pharmacists into the communication and interpretation of molecular rapid diagnostic tests (mRDT) for bloodstream infections and summarize outcomes from a 12-month post-implementation assessment. Physician and pharmacist impressions of the service are also described.

Summary: mRDT have proven clinical benefit in the treatment of bacteremia. Pharmacy leadership can collaborate with other health system leaders to develop policies and a workflow that route result calls to pharmacists to maximize the impact of this technology. Pharmacist education, development of clinical resources and documentation templates allow all pharmacists to perform this antimicrobial stewardship service consistently and confidently. Physicians overwhelmingly recognize the value of this service and often accept the pharmacist's recommendations. Antibiotic de-escalation was the most frequent outcome when changes to the antibiotic regimen were made.

Conclusion: Pharmacists are well positioned to utilize results from mRDT to improve antibiotic selection. Through the use of competencies and internally-derived resources, all pharmacists, rather than just infectious diseases pharmacy specialists, can perform this important antibiotic stewardship activity and positively influence patient outcomes.

Keywords: Molecular rapid diagnostic test, bacteremia, antimicrobial stewardship, sepsis, clinical pharmacy service

\section{BACKGROUND}

Bloodstream infections (BSI) are associated with significant increases in morbidity, mortality, and hospital lengths of stay. ${ }^{1}$ Delayed identification of the infecting pathogen and its associated resistance markers may be associated with prolonged exposure to suboptimal or inadequate antimicrobial therapy. Previous literature has established that delayed administration of effective antimicrobials increases mortality in septic patients with each hour of delay over the first six hours resulting in a $7.6 \%$ decrease in survival for these patients. ${ }^{2}$

Traditional pathogen culture and antimicrobial sensitivities usually require at least 48-96 hours before final results are made available. The National Action Plan for Combating Antibiotic Resistance and Infectious Diseases Society of America both support implementing molecular rapid diagnostic testing (mRDT) across our nation's healthcare settings to shorten the time to identifying bacteremia (Figure 1)..$^{3,4}$

There are multiple mRDT technologies available with differences in the organisms they can identify, ability to detect resistance markers and/or susceptibilities, and time to result. The 2017 Timbrook and colleagues publication in Clinical Infectious Diseases provides a thorough review and Table 1 offers a high-level comparison.

Corresponding author: Andrew Ticcioni, PharmD

Ascension Columbia St. Mary's Hospital

2323 N Lake Drive, Milwaukee, WI 53211

Email: Andrew.Ticcioni@Ascension.org
While there are significant costs to implementing and maintaining mRDT that may cause hospital administrators to question its value, multiple studies have demonstrated persuasive clinical outcomes.

Avdic and colleagues compared the optimization of antibiotics before and after the implementation of mRDT for Grampositive blood culture technology with antimicrobial stewardship interventions and demonstrated that patints with methicillin-susceptible Staphylococcus aureus received optimal antimicrobial optimal antibacterial therapy more than 30 hours sooner. ${ }^{5}$

A meta-analysis which included 31 studies and 5920 patients concluded mRDTs working in conjunction with an established antimicrobial stewardship program were associated with significant decreases in mortality, time to effective antimicrobial therapy, and hospital length of stay. ${ }^{1}$

The effects of implementing mRDT with a workflow that incorporated community hospital pharmacists resulted in a significant decrease in hospital length of stay, antimicrobial durations of therapy, improved time to targeted antimicrobial therapy, and hospital cost savings of around $\$ 7,240$ per patient. $^{6}$

Other studies and review articles demonstrated similar findings regarding the benefit of implementing any of the different mRDT commercially available modalities and recommend combined mRDT with antimicrobial stewardship intervention to be the standard of care for BSI. ${ }^{7,8,9}$ 
In addition to cost, another obstacle that prevents implementation of mRDT includes limited knowledge and understanding of how to integrate this technology within a hospital system. ${ }^{7}$

\section{INTRODUCTION}

The Centers for Disease Control and Prevention (CDC) identifies seven core elements of a hospital antimicrobial stewardship program. ${ }^{10}$ Pharmacy expertise, implementation of initiatives to improve antibiotic use, and tracking antibiotic use and outcomes are three of the seven core requirements. There has been steady improvement in the percentage of facilities meeting all seven core elements. In fact, the 2020 CDC update reported that $88.9 \%$ of hospitals met all seven core requirements. ${ }^{11}$ Interestingly, although there has been consistent increase in achievement of CDC core elements and growth in the number of hospitals with a dedicated antimicrobial stewardship pharmacist, fewer than $50 \%$ of hospitals have one. ${ }^{12}$ Instead, many hospitals rely on a more distributed accountability model for daily antimicrobial stewardship activities by utilizing pharmacists without specialty training in infectious diseases. ${ }^{13}$ For example, clinical staff pharmacists may incorporate antimicrobial stewardship services into their daily work though the prospective order review process, daily antibiotic usage reports, and possibly clinical surveillance software. It is important to equip these pharmacists with the training and resources to perform robust antimicrobial stewardship services. A survey of clinical pharmacists participating in antimicrobial stewardship programs showed that about half of respondents receive mRDT results in real time. ${ }^{14}$ As previously described, prompt initiation of antibiotics in patients with bacteremia has profound impact on mortality. Facilities with mRDT capability should have workflows in place to ensure the findings from this powerful tool are quickly reviewed by the patient's care team to optimize antibiotic selection and dosage.

\section{PURPOSE}

Here we describe how a 24-hour service was developed that includes all community hospital-based pharmacists in the communication of mRDT results for bloodstream infections and equips them with the knowledge and resources to make antibiotic therapy recommendations to prescribers. This service maximizes the utility of MRDT because all hospital pharmacists participate in receiving and responding to results as soon as they are known, and pharmacists formulate treatment recommendations based on facility-specific antibiotic resources. Readers will be provided with the stepwise process to successfully design and implement this type of antimicrobial stewardship service. A 12-month postimplementation assessment of all mRDT results at two hospital campuses was performed to characterize the service and associated outcomes. Surveys were distributed to pharmacists and physicians to gather their impressions of the mRDT notification service.

\section{SERVICE OVERVIEW}

The objective of this service is to include all staff pharmacists in the notification sequence when the Laboratory has positive mRDT results detecting a potentially pathogenic organism in the blood. Upon receiving the call with pathogen identification and resistance markers, the pharmacist assesses the patient's current antibiotic regimen (if one is present) and uses facilityspecific resources to determine if changes are needed to ensure appropriate antibiotic coverage until sensitivities are known. The pharmacist is responsible for promptly contacting the patient's physician to communicate the MRDT results and then collaboratively formulate an antibiotic treatment plan. Next, the pharmacist documents the mRDT results, current antibiotic regimen, and any changes to the antibiotic regimen in the electronic medical record (EMR). Pharmacists are also accountable for entering or modifying antibiotic orders in the EMR. This service is provided by all pharmacists, 24 hours a day, seven days a week. Figure 2 below summarizes the workflow from start to finish. Individual steps will be described in detail in subsequent sections.

Currently more than 120 pharmacists working on all shifts across seven hospitals provide this service as part of a clinical generalist practice model. The hospitals range in size from 50 to 300 beds and they all have onsite pharmacist staffing coverage 24 hours a day. Pharmacists across the system were already providing a variety of clinical services 24 hours a day, seven days a week (e.g. IV to PO conversions, dose adjustments for kidney function, aminoglycoside, vancomycin, anticoagulant, antiepileptic medication dosing and monitoring), responding to medical emergency and stroke response, attending critical care rounds, etc. The addition of a real time mRDT result interpretation as an additional antimicrobial stewardship activity was a natural extension of the Pharmacy Department services.

This service was developed and launched across seven hospitals in the Milwaukee area in two phases using Verigene ${ }^{\circledR}$ as the mRDT for positive blood cultures. The first phase took place at hospital campuses where new mRDT technology was added to the Microbiology Lab. The second phase was at hospital campuses already with mRDT, but the Microbiology Lab was only communicating results to the patient's nurse. The suggested procedure outlined below can be applied in either situation to direct mRDT results to the pharmacist.

\section{SERVICE BUILD \\ Engage stakeholders}

Design of a mRDT pharmacist notification service requires interdisciplinary support and coordination. Engage leadership from each department/workgroup to learn about the current workflow and policies for mRDT result reporting. Of course, prior to discussing a change in the existing process, Pharmacy leadership should determine if this service can be incorporated into the existing department functions. Although many of these 
discussions may occur simultaneously, below is a proposed sequence and associated area of focus for each entity.

\section{Pharmacy Department}

A careful assessment of the pharmacists' interest, aptitude, and time allowance should be performed prior to integrating them into the mRDT communication sequence. Most pharmacists will quickly realize the benefit of early organism identification provided by mRDT. Including a competency, antibiotic action plans, and tip sheet (all discussed later) as part of the service launch should provide the needed confidence and support to take on this responsibility. Pharmacy Department leadership will need to assess their practice model to identify which pharmacists will take mRDT results calls. Additionally, Pharmacy Department leadership will need to determine what committee approvals are necessary to integrate pharmacists into the mRDT result reporting process.

\section{Antimicrobial Stewardship Committee}

Changes to the mRDT communication service has broadreaching implications and will impact numerous disciplines and likely multiple hospital campuses. A reasonable starting point is to present a brief overview of the current communication sequence in use to the Antimicrobial Stewardship Committee. Summarize the benefits of adding a pharmacist into the communication sequence and describe how pharmacists will be trained to reliably perform this new service. If the Antimicrobial Stewardship Committee agrees, consider setting up an interdisciplinary workgroup with representatives from the departments listed below. Alternatively, a member of the pharmacy department could coordinate this work outside the committee.

3. Laboratory

The Laboratory Department and its leadership will be an integral part of implementing a new communication sequence. Identify a member of Laboratory leadership from the Microbiology Department. This individual may already be a member of the Antimicrobial Stewardship Committee. It is possible this will be the first large project coordinated between the Microbiology Lab and Pharmacy Department. First, determine what type of mRDT devices are in use for positive blood cultures. It is important to determine if both Gram-positive and Gram-negative results are analyzed, what organisms are identified, and if resistance markers are detected. Together, map out the current result notification sequence and associated policies and procedures for these results. If possible, quantify the number of results reported by getting a daily or weekly average based on a 3-6 month timeframe. Ensure the pharmacy department can accommodate this call volume, complete an antibiotic review, and notify the provider. Obtain copies of the exact result outputs from the mRDT device(s) and what verbiage the Laboratory staff use to communicate the results over the phone and in the medical record. Keep the Laboratory leadership updated on the progress of the service development and planned go live date. Additionally, following implementation take note of feedback from front-line clinical staff; this feedback may be vital for ongoing process improvements.

\section{Physicians}

A variety of physician groups should be included in the review and approval of this notification service. Specifically, the hospitalist group, intensivist / pulmonologist group for critical care consults, medical residency program leadership, the infectious diseases group, and any contracted or private physician organizations privileged for acute care services. If results for discharged patients are included in the service, the emergency medicine provider group should also be involved since some patients who were discharged from the emergency department may have positive results. Additionally, the antibiotic recommendations in any resources should be vetted through these physician and prescriber groups.

\section{Nursing}

Most critical laboratory result notification calls, such as positive blood culture identification/susceptibility or positive blood culture Gram-stains, are received by nurses. Oftentimes, hospital policy requires the nurse to pass the critical result notification onto the patient's attending physician. Utilizing pharmacists in place of nurses presents an opportunity to engage physicians in antibiotic therapy discussions based on the results rather than just communicating them. Nursing leadership and any leadership councils or workgroups should be included in a proposal to have a pharmacist receive mRDT results. Similar to what was done with the Microbiology Lab, review established policies and procedures for communication and documentation of critical laboratory results and identify any adaptations necessary as a result of having a pharmacist receive the MRDT call instead of or in addition to the nurse. Finally, ensure proper isolation/contact/droplet precautions will be maintained depending on the organism(s) identified if the communication sequence is changed.

\section{Hospital Administration}

Leadership from hospital administration may prove beneficial for a number of reasons. Laboratory equipment that performs mRDT is costly to purchase, operate, and maintain. For example, a site adding polymerase chain reaction mRDT could expect approximately $\$ 30,000$ in startup hardware costs, $\$ 10,000$ in annual maintenance fees, the cost for each test and Laboratory staff time to perform and report the test, plus the cost of any repairs. These prices are estimates and would depend on the number of anticipated analyses. If microbiology services are not centralized, multiple readers and processors would be required. If your facility or health system considers purchasing mRDT equipment, or has previously denied it, inclusion of a pharmacist in the results sequence has a proven benefit and may be a motivating factor to obtain 
this technology. Hospital administration should also be made aware of how pharmacists could work to improve clinical outcomes and antibiotic utilization by receiving mRDT results and promptly collaborating with providers to formulate a treatment plan. Lastly, engaging hospital administration early may assist with getting the support of other disciplines and also facilitate any necessary changes to established policies and procedures.

\section{Develop Resources}

Internal resources are foundational to the successful launch and daily operation of this service. Pharmacy students and residents are well suited to assist in the creation of these tools. An anticipated time period of 3-6 months should be allotted to design these tools, deploy education, and initiate this service. Certain approvals may require additional time.

\section{Antibiotic Action Plans}

Development of detailed resources allows all pharmacists to provide the service. Specifically, "Antibiotic Action Plans" facilitate proper antimicrobial recommendations based on the pathogen and resistance markers identified and provide consistency in treatment recommendations. The Action Plans should be readily, concise, and user-friendly for pharmacists. For example, the Action Plans could be formatted as a table with the exact output information the Laboratory provides when communicating the results to the pharmacist (Figure 3). For each organism and resistance marker, the Action Plan provides the drug of choice and suitable alternatives. An Action Plan for each Gram-positive and Gram-negative organism may be appropriate depending on what mRDT equipment is in use. An overview of general service expectations should be included in the document along with the table of "bug-drug" recommendations. Action Plans should be customized to each site utilizing the facility formulary and local antibiogram to ensure proper coverage. Recommended doses and dose adjustments along with alternative agents in the event of allergies or other contraindications could also be included. Once drafted, Action Plans should be reviewed and approved by infectious diseases physicians in order to ensure agreement on optimal antibiotic recommendations. When finalized, the Action Plans may be vetted through the Pharmacy \& Therapeutics Committee for approval and distributed amongst pharmacists. Routine review of the antibiotic recommendations in Action Plans should coincide with the results of the annual antibiogram. General guidance and expectations may be placed on the Action Plan references so users understand how to perform the service and use the reference.

\section{Standardized Documentation}

Documentation of mRDT results may already be part of an existing policy or procedure. Laboratory staff will likely record the mRDT result directly on the blood culture result where sensitivities will ultimately be posted. Review established documentation standards with Laboratory and Nursing leadership and determine what documentation the pharmacist should perform as part of the result communication sequence. Bacteremia is a serious clinical finding. Creation of a standardized note template in the EMR saves time and ensures consistency in documentation (Figure 4). The pharmacist should document the mRDT result, current antibiotic regimen (if any), the prescriber who was notified of the results, the date and time of the prescriber notification, and if orders were received to make changes to the antimicrobial treatment.

\section{Prepare Pharmacists}

Pharmacist Training, Competency, Tip Sheet, and Antimicrobial Stewardship Team:

Any pharmacist who will receive mRDT result calls should receive training on the technology, resources, and service expectations. Training may be provided through department inservices, web-based modules, recorded presentations, or printed materials. Training can be self-paced and generally completed in less than two hours. Following the training, a competency may be deployed to ensure comprehension. Elements of a pharmacist competency should include the intended scope of mRDT results and limitations. Specifically, the competency should address specimen collection, types of identifiable resistance markers and timing sequence between specimen collection and mRDT results. Pharmacists should be aware of scenarios when antibiotic de-escalation may not be warranted such as polymicrobial infections (e.g. diabetic foot ulcers, intra-abdominal infections, etc.), infections where source control has not been achieved, and in settings where multidrug resistant organisms are suspected. Competency questions using clinical cases in application-based questions adds to pharmacist comprehension and confidence. Antibiotic Action Plans should be used in the competency as part of the cases so pharmacists develop familiarity with how to use them. Additionally, the competency should address established communication workflow between disciplines as well as documentation requirements for mRDT results, interpretation, timing of physician contact, and action taken as a result of collaborative discussion with physician. Consider discussing the results of the competency with the department, specifically, what questions were commonly answered incorrectly. Pharmacists could even be provided with an answer key that includes the rational for each correct response. New pharmacists, including residents, should be required to complete the training and competency prior to providing the service.

Creation of a tip sheet for quick reference will assist pharmacists in providing the service upon receipt of a mRDT result call from the Laboratory. A tip sheet should outline the expected workflow, documentation, and communication pathway the pharmacist will be engaging in each time they receive a result from mRDT. Additionally, this resource provides the pharmacist with the clinical expectations associated with each mRDT result, including which aspects of the patient's chart are pertinent to review when deciding if an antimicrobial 
change is warranted, a list of organisms and resistance markers the mRDT can detect, how to interpret less clear clinical scenarios (e.g. polymicrobial infections, blood culture contamination versus true bloodstream infection with coagulase-negative Staphylococci), and which scenarios are crucial for pharmacists to be recommending an infectious diseases consult to the primary team.

Lastly, for sites with a dedicated antimicrobial stewardship team/pharmacist, in addition to developing resource materials and determining clinical expectations of the staff pharmacists, hosting roundtable discussions with staff pharmacists prior to service launch allows for face-to-face education and solicitation of feedback. Additionally, it is important staff pharmacists know how to reach the antimicrobial stewardship team/pharmacist when they encounter clinical scenarios not addressed in the antibiotic action plans and other resource documents. Contact information can be listed in the tip sheet and other associated resource documents.

\section{Equip the Laboratory}

Map the Communication Sequence:

Provide the Laboratory with direct contact information for each pharmacist service/team so whenever possible, result calls can be routed directly to the pharmacist caring for that patient. Laboratory staff may need to call the Central Pharmacy if results need to be communicated when pharmacy services are centralized. Some microbiology services in health systems with multiple campuses operate from a centralized laboratory. Determine where blood samples are sent for culture and mRDT analysis so the Laboratory can reach a pharmacist at the correct facility who will provide the service and notify the provider.

\section{SERVICE LAUNCH}

An official memo should be distributed to facility medical staff and nursing leaders prior to the service launch summarizing the new mRDT result communication sequence, pharmacist response, and weblinks to the antibiotic action plans along with the go-live date. Nursing leaders should cascade the information contained in the memo with staff nurses so that front line care givers are aware of any changes and who is accountable for the critical lab result. A copy of the same memo should be provided to the pharmacists. Pharmacists should also be reminded of the upcoming go-live date during any department meetings or huddles.

Following implementation of the new communication service, Pharmacy leaders should solicit feedback from pharmacists on the new service. Keeping an open communication channel is crucial for process improvement and troubleshooting any aspect that might be unclear or unique scenarios not considered prior to implementation. Additionally, individual pharmacists can be interviewed by leadership during monthly associate rounding to learn about their personal experience with the new service. Generalizable information from these conversations could be shared at department meetings or via email.

It is helpful to audit the service during the first few months to ensure positive mRDT results are being routed to pharmacists and the proper actions, including documentation, are being performed. For sites with an antimicrobial stewardship team/pharmacist, solicit feedback on a regular basis during the first few months when rounding with Pharmacy leaders, staff pharmacists, and Microbiology staff.

\section{SERVICE OUTCOMES Methods}

A 12-month post-implementation retrospective chart review was conducted at the two most recent hospitals to launch the mRDT pharmacist interpretation and communication service. This investigation was granted exemption from formal review by the institutional review board. One hospital is an urban community teaching hospital in Milwaukee, Wisconsin with 36 intensive care beds, a 12-bed regional burn center, and an average daily census of 240 patients. The second hospital is a suburban community hospital located in Mequon, Wisconsin with 32 adaptable acuity beds and an average daily census of 80 patients. All hospitalized patients with a mRDT result from April 2019 to March of 2020 were included in the review. Results for patients treated and discharged from the Emergency Department were excluded since these were not in scope for the service.

\section{Results}

916 patients with mRDT results were evaluated. Eighty-one mRDT results were excluded because they were from Emergency Department patients who were not admitted to the hospital. The remaining 835 were reviewed. Five-hundred and ninety-four $(71 \%)$ results were from the Milwaukee Campus and 251 (29\%) were from the Mequon Campus. Findings are summarized in Table 2 . Ninety point eight percent $(90.8 \%)$ of the patients were receiving antimicrobials prior to the mRDT result. Thirty-three point four percent (33.4\%) of patients had a change in their antimicrobial regimen upon receipt of the MRDT results and recommendation from the pharmacist. Gramnegative $\mathrm{mRDT}$ had antibiotic regimen changes more often than Gram-positive. A change was referred to as either an initiation, addition, discontinuation, or change in dose, or frequency of the current antimicrobial regimen. As shown in Figure 5, the reasons for antimicrobial changes following pharmacist communication of mRDT results were 1) deescalation to a narrower spectrum agent (74.9\%), 2) increased dosing to match indication (17.2\%), 3) broadening of coverage (4.7\%), followed by 5) discontinuation of therapy (3.2\%). Grampositive mRDT results were much more likely to have a resistance marker identified than Gram-negative results. 


\section{Pharmacist and Physician Impressions Pharmacists}

A 12-question, multiple choice survey was distributed to all pharmacists at the two hospitals 15 months after the mRDT pharmacist interpretation and communication service was implemented. Many questions used the traditional 5-point Likert scale. All three shifts and the pharmacy residents received the survey. Fifty-six percent (35/62) of pharmacists completed the survey.

Prior to the implementation of the mRDT service, $45.7 \%$ of pharmacists reported routinely including mRDT results in their antimicrobial stewardship practice activities. The competency and tip sheet were identified as the most helpful training resource to perform the service. A majority of the pharmacists $(88.6 \%)$ agreed or strongly agreed that the antibiotic action plans are helpful. When asked how long it takes to perform the mRDT interpretation and communication service, $51 \%$ of pharmacists reported it can be done in 11-20 minutes while $25 \%$ of pharmacists do it in 5-10 minutes. Nearly all respondents $(88.5 \%)$ rated themselves as confident when making antibiotic recommendations to physicians based on a mRDT result. Overall, $68.6 \%$ pharmacists believe the service improves patient care and $51.5 \%$ believe it saved them time overall with respect to general antimicrobial stewardship practice expectations.

\section{Physicians}

A 9-question, multiple choice survey was distributed to the intensivist and hospitalist teams at the same two hospitals 15 months after implementation of the mRDT pharmacist interpretation and communication service. Surgeons and specialists (including infectious diseases physicians) did not receive the survey since most mRDT results are reported early in the hospitalization often before these parties are consulted. Medical residents, faculty and locum physicians did not receive the survey because they were likely not on staff before and after the service launch to comment on the comparison. Nearly all of the questions used the traditional 5-point Likert scale. The survey had an overall response rate of $22 \%$ (9/41) with $21 \%$ (7/33) of the hospitalists and $25 \%(2 / 8)$ of intensivists participating, respectively.

Physicians unanimously agreed or strongly agreed that the pharmacists are confident when providing antibiotic recommendations and that the recommendations are helpful. Nearly all physicians (8/9) usually accept the antibiotic recommendations offered. When asked if the mRDT result calls from pharmacists improve patient care, 67\% (6/9) strongly agreed, $11 \%$ (1/9) agreed and 22\% (2/9) had a neutral response. Respondents had a very mixed response when asked if the pharmacist's recommendation to obtain an infectious diseases consult for Gram-positive mRDT result was appreciated. Prior to the pharmacist mRDT result service launch, the patient's nurse communicated mRDT results to the physician. When asked if the physicians preferred mRDT results from the pharmacist or nurse they all preferred the pharmacist providing the notification.

\section{DISCUSSION}

The incorporation of pharmacists into the mRDT reporting workflow by contacting prescribers directly with antimicrobial recommendations allows for this collaborative discussion promptly after results are known. Utilization of all pharmacists rather than infectious diseases specialists allows this service to be performed 24 hours a day, seven days a week without delay.

Implementation of $\mathrm{mRDT}$ result communication with pharmacist intervention requires a detailed plan involving leadership from all departments that will be affected by this service. A self-paced training program followed by a competency, antibiotic action plans, and tip sheet equipped our team of clinical generalists well to perform this new service.

Past reviews and meta-analyses have demonstrated decreased mortality, time spent within the hospital, and a reduction in median time to antimicrobial de-escalation from empiric therapy for patients when a mRDT and antimicrobial stewardship team are utilized together. ${ }^{1,3}$ The assessment of our service showed that antibiotic de-escalation was a common outcome.

Each hospital campus averaged one to two result calls per day over the 12-month assessment time period. Pharmacists generally perform the service from start to finish in less than 20 minutes. This is a manageable amount of time for this critical, high-impact intervention.

Survey response rate was low among physicians, but those who responded overwhelmingly reported that the staff pharmacists are confident, their recommendations are helpful, and the service creates a perceived improvement in patient outcomes.

Readers should keep in mind that this is a descriptive report of experiences at two hospitals over a short time period. Outcomes may be different at other facilities.

\section{CONCLUSION}

Past literature has demonstrated mRDTs have the ability to be powerful antimicrobial stewardship tools, especially in the setting of bloodstream infection management. Timely incorporation of pharmacists in the communication and clinical application of results from mRDTs is essential for maximizing the potential of these tools. Careful and considered planning is necessary for a successful mRDT service launch. This report outlined our stepwise approach to implementing this 24/7 service across our entire pharmacist workforce with a demonstrated impact on patient care and physician satisfaction. 
Acknowledgements: Ascension Wisconsin Laboratory staff, with special appreciation for Sarah Cliff, MLS (ASCP) and Eric Lenz, MT (ASCP), the Antimicrobial Stewardship Program Committee and physician leaders, Infection Prevention, and Nursing leadership

\section{References}

1. Timbrook TT, Morton JB, McConeghy KW et al. The effect of molecular rapid diagnostic testing on clinical outcomes in bloodstream infections: a systematic review and meta-analysis. Clin Infect Dis. 2017; 64: 1523.

2. Kumar A, Roberts D, Wood KE et al. Duration of hypotension before initiation of effective antimicrobial therapy is the critical determinant of survival in human septic shock. Crit Care Med. 2006; 34:1589-96.

3. U.S. Department of Health and Human Services. National action plan for combating antibiotic-resistant bacteria (October 2017). https://aspe.hhs.gov/system/files/pdf/258516/Progres sYears1and2CARBNationalActionPlan.pdf (accessed 2020 April 27).

4. Barlam TF, Cosgrove SE, Abbo LM et al. Implementing an antibiotic stewardship program: guidelines by the Infectious Diseases Society of America and the Society for Healthcare Epidemiology of America. Clin Infect Dis. 2016; 62: e51-77.

5. Avdic E, Wang R, Li DX et al. Sustained impact of a rapid microarray-based assay with antimicrobial stewardship interventions on optimizing therapy in patients with gram-positive bacteremia. J Antimicrob Chemother. 2017; 72: 3191-98.

6. Box MJ, Sullivan EL, Ortwine KN et al. Outcomes of rapid identification for gram-positive bacteremia in combination with antibiotic stewardship at a community-based hospital system. Pharmacotherapy. 2015; 35: 269-76.
7. Timbrook TT, Wenzler E. The current state of antibiotic stewardship and rapid diagnostic testing. Infectious Disease Special Edition. 2019: 42-46.

8. Wenzler E, Timbrook TT, Wong JR, Hurst JM et al. Implementation and optimization of molecular rapid diagnostic tests for bloodstream infections. Am J Health Syst Pharm. 2018; 75: 1191-1202.

9. Felsenstein S, Bender JM, Sposto R, et al. Impact of a rapid blood culture assay for Gram-positive identification and detection of resistance markers in a pediatric hospital. Arch Pathol Lab Med. 2016; 140(3): 267-75.

10. Centers for Disease Control and Prevention. Core Elements of Hospital Antibiotic Stewardship Programs. Atlanta, GA: US Department of Health and Human Services, CDC; 2019. Available at https://www.cdc.gov/antibiotic-use/coreelements/hospital.html. Accessed March 28, 2021.

11. Centers for Disease Control and Prevention. Antibiotic Use in the United States, 2020 Update: Progress and Opportunities. Atlanta, GA: US Department of Health and Human Services, CDC; 2020. Available at https://www.cdc.gov/antibiotic-use/stewardshipreport/current.html. Accessed March 28,2021.

12. Pederson CA, Schneider PJ, Ganio MC, Scheckelhoff DJ. ASHP national survey of pharmacy practice in hospital settings: monitoring and patient education-2018. AM J Health-Syst Pharm. 2019; 76: 1038-58.

13. Collins, CD, Miller DE, Kenney RM, et al. The state of antimicrobial stewardship in Michigan: results of a statewide survey on antimicrobial stewardship efforts in acute care hospitals. Hosp Pharm. 2015; 50:80-184

14. Foster RA, Kuper, K, Lu ZK, et al. Pharmacists' familiarity with and institutional utilization of rapid diagnostic technologies for antimicrobial stewardship. Infect Control Hosp Epidemiol. 2017; 38:863-866. 


\section{Blood Sample \\ Collection \\ $\mathrm{T}=0$ hours}

mRDT Result

$T=$ Within 3 hours of

positive Gram stain
Traditional

Identification and

Sensitivities

$\mathrm{T}=48-96$ hours

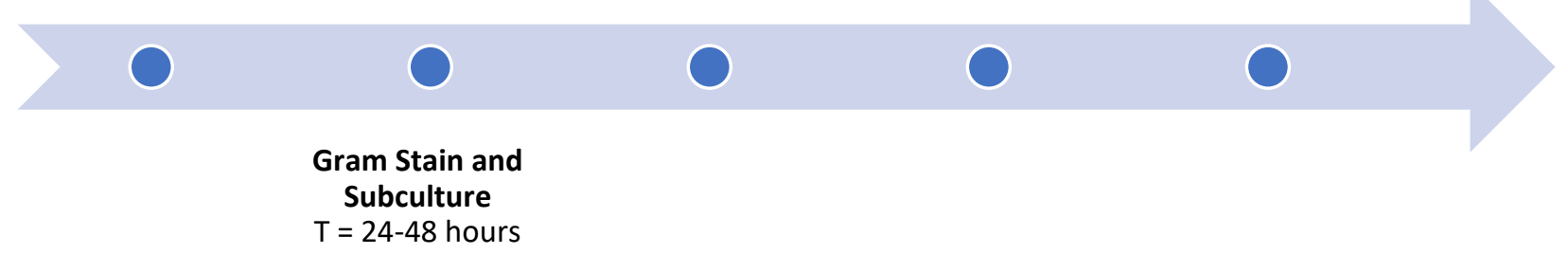

Figure 1: Sample Blood Culture Reporting Sequence with PCR mRDT ${ }^{1}$

$\mathrm{T}=$ time, $\mathrm{PCR}=$ polymerase chain reaction, $\mathrm{mRDT}=$ molecular rapid diagnostic testing

Table 1: Available mRDT Characteristics

\begin{tabular}{|c|c|c|c|}
\hline mRDT Technology & Approximate Time to Result* & $\begin{array}{c}\text { Resistance Marker/ } \\
\text { Susceptibility Detection }\end{array}$ & Trade Names \\
\hline MALDI-TOF MS & 40 hours & No & MALDI Biotypr $^{\circledR}$ \\
\hline Magnetic resonance & $3-5$ hours & No & T2Bacteria $^{\circledR}$ Panel \\
\hline PNA-FISH & $24-30$ hours & No & AdvanDx QuickFISH $^{\circledR}$, PNAFISH $^{\circledR}$ \\
\hline PNA-FISH MCA & $24-30$ hours & Yes & Accelerate PhentoTest $^{\circledR}$ \\
\hline Polymerase chain reaction & $24-30$ hours & Yes & BioFire $^{\circledR}$, Verigene $^{\circledR}$ \\
\hline
\end{tabular}

mRDT = molecular rapid diagnostic testing, MALDI-TOF MS= matrix-assisted laser desorption/ionization-time of flight mass

spectrometry, PNA-FISH = peptide nucleic acid fluorescent in situ hybridization, MCA = morphokinetic cellular analysis

*From start of sample analysis

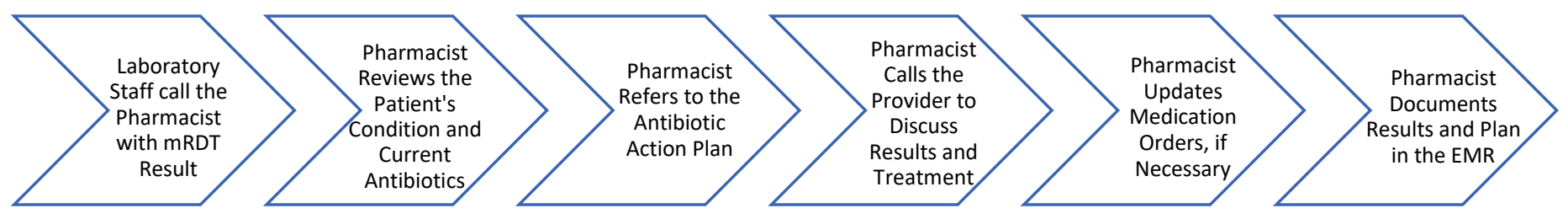

Figure 2: Molecular Rapid Diagnostic Test Result Workflow

$\mathrm{mRDT}=$ molecular rapid diagnostic testing, $\mathrm{EMR}=$ electronic medical record 


\section{Pharmacy Rapid Diagnostic Blood Test - Gram Negative Antibiotic Action Plan}

Verigene ${ }^{\circledR}$ is a commercial rapid diagnostic tool that uses molecular detection technology to identity pathogens and resistance markers in blood samples.

- $\quad$ Pharmacists will use the table below as guidance to facilitate prompt antibiotic selection for patients with positive blood cultures. The recommendations included in this action plan are only for bloodstream infections; other infections with concomitant bacteremia should be treated according to best practice recommendations. Other considerations should include a patient's clinical status, source of infection, evidence of prosthetic implant(s), and history of multi-drug resistant organisms.

- $\quad$ Orders must be obtained from a provider for any antibiotic regimen changes.

- $\quad$ Pharmacists must document the Verigene results and treatment plan in the medical record.

- A provider must be notified of the confirmed bacteremia, even if the patient is already receiving the drug of choice.

\begin{tabular}{|c|c|c|c|c|c|}
\hline \multicolumn{6}{|c|}{ Gram Negative Rod: Fermenters } \\
\hline Organism & \multicolumn{2}{|c|}{ Resistance Marker } & Action Plan & Drug of Choice (DoC)* & Alternate Drug* \\
\hline \multirow{2}{*}{$\begin{array}{l}\text { Escherichia coli } \\
\text { Proteus spp. } \\
\text { Klebsiella } \\
\text { pneumoniae } \\
\text { Klebsiella oxytoca }\end{array}$} & $-\mathrm{CTX}-\mathrm{M}$ & & $\begin{array}{l}\text { Notify MD, de-escalate } \\
\text { to DOC if possible }\end{array}$ & Ceftriaxone $2 \mathrm{~g}$ q24h & $\begin{array}{l}\text { Cefepime } 1 \mathrm{~g} \text { q6h -OR- } \\
\text { Aztreonam } 2 \mathrm{~g} \text { q8h for patients } \\
\text { with a severe } \beta \text {-lactam allergy }\end{array}$ \\
\hline & $+\mathrm{CTX}-\mathrm{M}^{* *}$ & $\begin{array}{l}\text { ESBL- } \\
\text { Producer }\end{array}$ & $\begin{array}{l}\text { Notify MD to change } \\
\text { antimicrobial }\end{array}$ & Meropenem 500mg q6h & $* *$ \\
\hline \multirow[t]{2}{*}{ Citrobacter spp. } & $-\mathrm{CTX}-\mathrm{M}$ & & $\begin{array}{l}\text { Notify MD, de-escalate } \\
\text { to DOC if possible }\end{array}$ & $\begin{array}{l}\text { Cefepime } 1 \mathrm{~g} \text { q6h } \\
\text { (due to concern for AmpC } \\
\text { upregulation) }\end{array}$ & Meropenem 500mg q6h \\
\hline & $+\mathrm{CTX}-\mathrm{M}^{* *}$ & $\begin{array}{l}\text { ESBL- } \\
\text { Producer }\end{array}$ & $\begin{array}{l}\text { Notify MD to change } \\
\text { antimicrobial }\end{array}$ & Meropenem 500mg q6h & $* *$ \\
\hline \multirow[t]{2}{*}{ Enterobacter spp. } & $-\mathrm{CTX}-\mathrm{M}^{* *}$ & & $\begin{array}{l}\text { Notify MD, de-escalate } \\
\text { to DOC if possible }\end{array}$ & $\begin{array}{l}\text { Cefepime } 1 \mathrm{~g} \text { q6h (due to } \\
\text { concern for AmpC up } \\
\text { regulation) }\end{array}$ & Meropenem 500mg q6h \\
\hline & $+\mathrm{CTX}-\mathrm{M}^{* *}$ & $\begin{array}{l}\text { ESBL- } \\
\text { Producer }\end{array}$ & $\begin{array}{l}\text { Notify MD to change } \\
\text { antimicrobial }\end{array}$ & Meropenem 500mg q6h & $* *$ \\
\hline
\end{tabular}

Figure 3: Sample Section of the Gram-negative Antibiotic Action Plan with Guidance (not all organism outputs shown) ESBL = extended-spectrum beta lactamase, ID = infectious diseases 


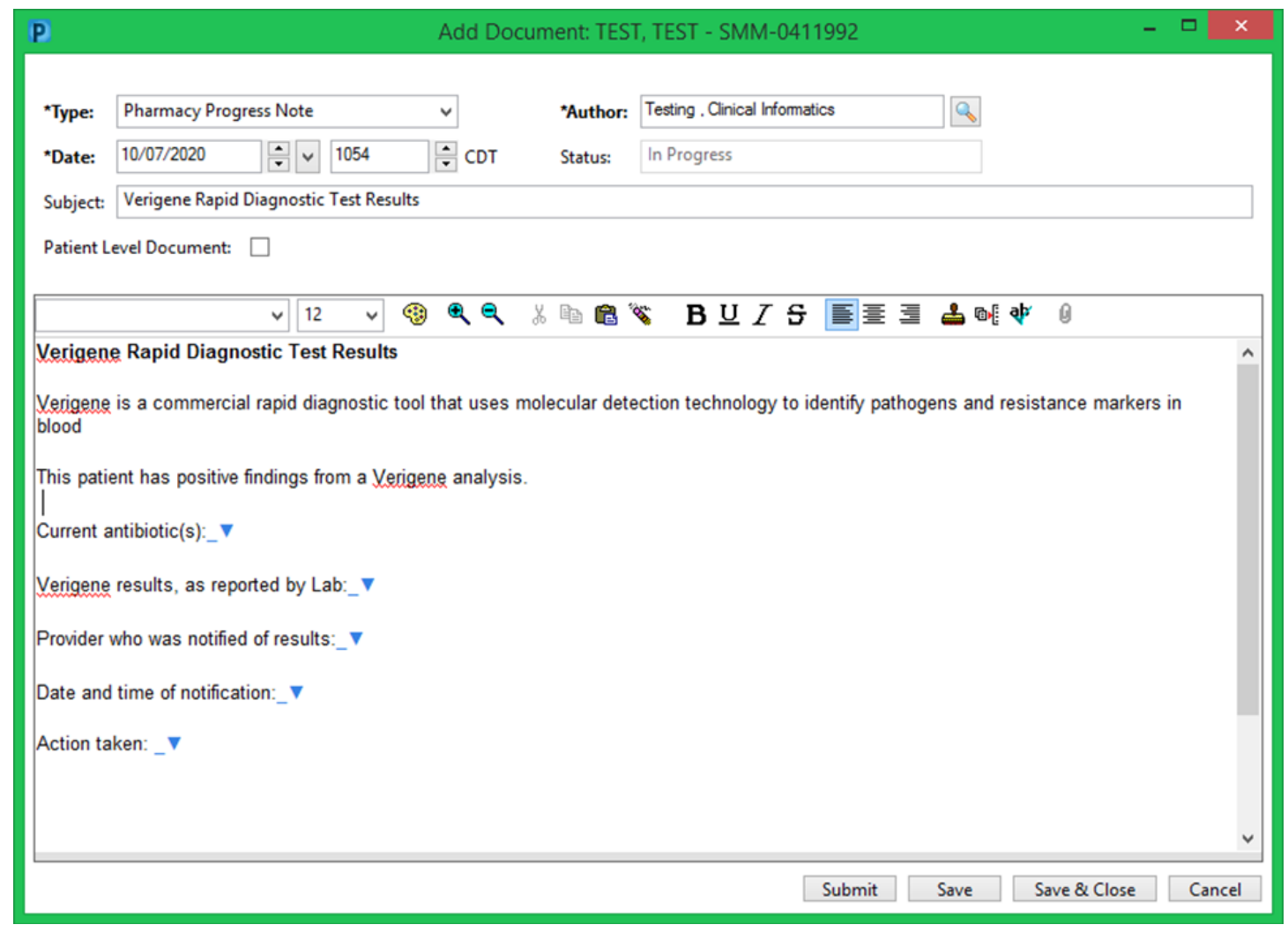

Figure 4: Sample mRDT result note template in the electronic medical record

Table 2: mRDT Outcomes Summary

\begin{tabular}{|c|c|c|c|}
\hline & Gram-positive organisms & Gram-negative organisms & Total \\
\hline Number of results, $\mathrm{n}(\%)$ & $577(69.1)$ & 258 (30.9) & 835 \\
\hline $\begin{array}{l}\text { Patient receiving antibiotics prior } \\
\text { to } \mathrm{mRDT} \text { result, } \mathrm{n}(\%)\end{array}$ & $\begin{array}{l}\text { Yes: } 504(87.3) \\
\text { No: } 73(12.7)\end{array}$ & $\begin{array}{l}\text { Yes: } 254(98.4) \\
\text { No: } 4(1.6)\end{array}$ & $\begin{array}{l}\text { Yes: } 758(90.8) \\
\text { No: } 77(9.2)\end{array}$ \\
\hline $\begin{array}{l}\text { Antibiotic regimen changed after } \\
\text { mRDT result call, } n(\%)\end{array}$ & $167(28.9)$ & $112(43.4)$ & $279(33.4)$ \\
\hline Change in antibiotic regimen, $\mathrm{n}(\%)$ & $\begin{array}{l}\text { Narrow coverage: } 141 \\
(84.4) \\
\text { Increased dose of current } \\
\text { therapy: } 10(6) \\
\text { Discontinued therapy: } 9 \\
\text { (5.4) } \\
\text { Broaden coverage: } 7(4.2)\end{array}$ & $\begin{array}{l}\text { Narrow coverage: } 68(60.7) \\
\text { Increased dose of current } \\
\text { therapy: } 38(33.9) \\
\text { Broaden coverage: } 6(5.4)\end{array}$ & $\begin{array}{l}\text { Narrow coverage: } 209 \text { (74.9) } \\
\text { Increased dose of current } \\
\text { therapy: } 48(17.2) \\
\text { Broaden coverage: } 13(4.7) \\
\text { Discontinued therapy: } 9 \text { (3.2) }\end{array}$ \\
\hline Resistance marker detected, $\mathrm{n}(\%)$ & $136(23.6)$ & $14(5.4)$ & $150(18)$ \\
\hline
\end{tabular}

$\mathrm{mRDT}=$ molecular rapid diagnostic testing 


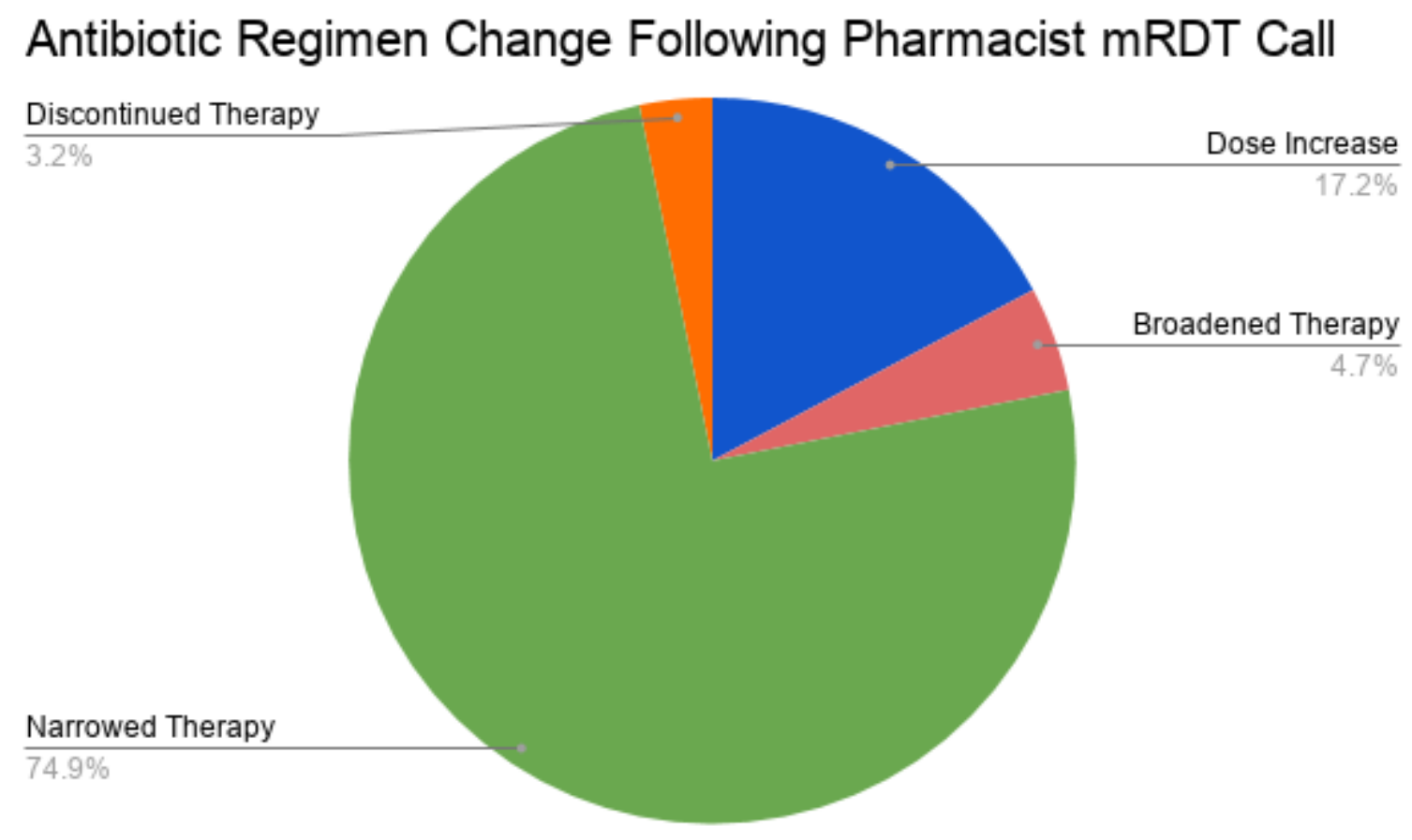

Figure 5: Antibiotic Regimen Change Following mRDT Call 